\title{
Bad germs are trapped
}

Cell Research (2018) 28:141-142. doi:10.1038/cr.2018.5; published online 9 January 2018

\begin{abstract}
Activation of the NAIP/NLRC4 inflammasomes initiates inflammatory responses against bacterial invasion. Two recent reports described the cryo-EM structure of the flagellin/ NAIP5 complex, providing important insights into the mechanism of bacterial sensing by innate immunity.
\end{abstract}

In the innate immune system, Tolllike receptor (TLR) pathways and inflammasome pathways activate inflammation. TLR pathways induce the production of pro-inflammatory cytokines, while inflammasome pathways control their activation and release. Inflammasomes are cytosolic protein complexes that activate caspase- 1 , which processes inflammatory cytokines such as pro-IL$1 \beta$ and pro-IL-18 and the pore forming protein gasdermin $\mathrm{D}$ into their functional forms, leading to cytokine release and pyroptotic cell death.

Cytosolic bacterial infection is restricted by the NAIP/NLRC4 inflammasomes. Both NAIP proteins and NLRC4 belong to the nucleotide-binding domain (NBD) and leucine-rich repeat (LRR) domain (NLR) protein family. Structurally, NAIPs and NLRC4 share a similar domain organization of NBD, helical domain 1 (HD1), winged helix domain (WHD), helical domain 2 (HD2), and LRR [1]. NAIPs have an additional Nterminal domain (NTD) and three baculovirus inhibitor of apoptosis protein repeat (BIR) domains before the NBD, and an insertion domain (ID) between HD2 and LRR. NLRC4 has an N-terminal caspase recruitment domain (CARD), which binds pro-caspase-1 CARD and ASC CARD, through CARD-CARD interactions. Different NAIPs sense different bacterial effector proteins, e.g., mouse
NAIP2 recognizes the rod protein of the type III secretion system, mouse NAIP5 and NAIP6 recognize flagellin, mouse NAIP1 recognizes the needle protein of the type III secretion system, and human NAIP (hNAIP) may recognize multiple ligands including rod protein, needle protein and flagellin [2-4].

The structure of NLRC4 activated by a ligand/NAIP complex was solved in $2015[5,6]$. Briefly, the ligand-bound NAIP2 activates the adaptor NLRC4 by converting its auto-inhibited closed conformation to an activated open state, and nucleates NLRC4 polymerization to mediate signal amplification and recruitment of caspase-1 [5, 6] (Figure 1A). Two recent papers published in Science by Tenthorey et al. [7] and in Cell Research by Yang et al. [8] reported the cryo-EM structure of an open conformation of NAIP5 bound to flagellin. Tenthorey et al. solved the structure of a NAIP5/flagellin complex together with two subunits of NLRC4 at 5.2 Å resolution, and Yang et al. solved a similar structure of the NAIP5/flagellin complex together with an oligomerization-deficient mutant of NLRC4 at $4.28 \AA$ resolution. These new structures provided a novel mechanism for the activation of NLR family proteins, and revealed important information on our understanding of bacterial recognition by inflammasome complexes.

Different from NLRC4 activation, which is promoted by the nucleating surface of NAIP5 or an activated NLRC4, the activation of NAIP5 is induced by binding of a bacterial ligand. After activation, both NAIP5 and NLRC4 expose the nucleating surface and recruit next NLRC4 molecule to transduce the signal (Figure 1A). The overall structure of NAIP5 is similar to that of NLRC4 because they share a similar domain organization. However, there are several unique features of NAIP5 at the NTD, BIR, HD2, ID and LRR domains, most of which participate in flagellin binding and allow NAIP5 to act as a receptor (Figure 1B). Flagellin is wrapped around by NAIP5 through multiple interaction sites and has no contact with NLRC4.

Both papers found that the $\mathrm{N}$-terminal helix (D0N), the C-terminal helix (D0C) and a connected fragment between D0N and D1 (hereafter named as D0N') of flagellin (Figure 1B) are bound to NAIP5 even though the two groups used flagellin from different bacteria and generated different constructs in their studies - Tenthorey et al. used the full-length Legionella flagellin FlaA, and Yang et al. used a truncated Salmonella flagellin FliC containing D0N, D0C and D0N' fragments. Both groups found that the D0C exerts extensive interaction with NAIP5, which plays the most important role in activating NAIP5 compared to the D0N and D0N' fragments. On NAIP5, the HD2, NTD, BIR1, ID and LRR domains form a deep pocket along the D0C helix and bind the ligand through both hydrophobic and hydrophilic interactions. Especially, three leucine residues of the D0C fragment are positioned into the hydrophobic pocket formed by NAIP5 NTD, BIR1 and HD1 (Figure 1B). Tenthorey et al. proposed that the extensive, multisurface recognition by NAIP5 and the function of the D0 domain in both flagellar filament formation and innate immune detection might limit flagellin evolution and pathogen immune evasion. In contrast, Yang et al. pointed 
A
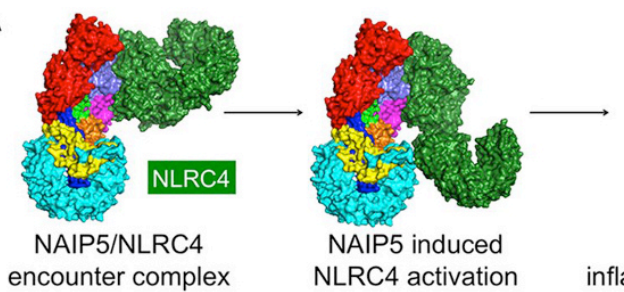

NAIP5 induced NLRC4 activation

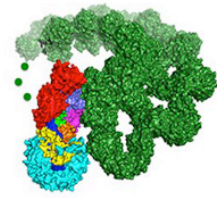

NAIP5/NLRC4 inflammasome formation
B

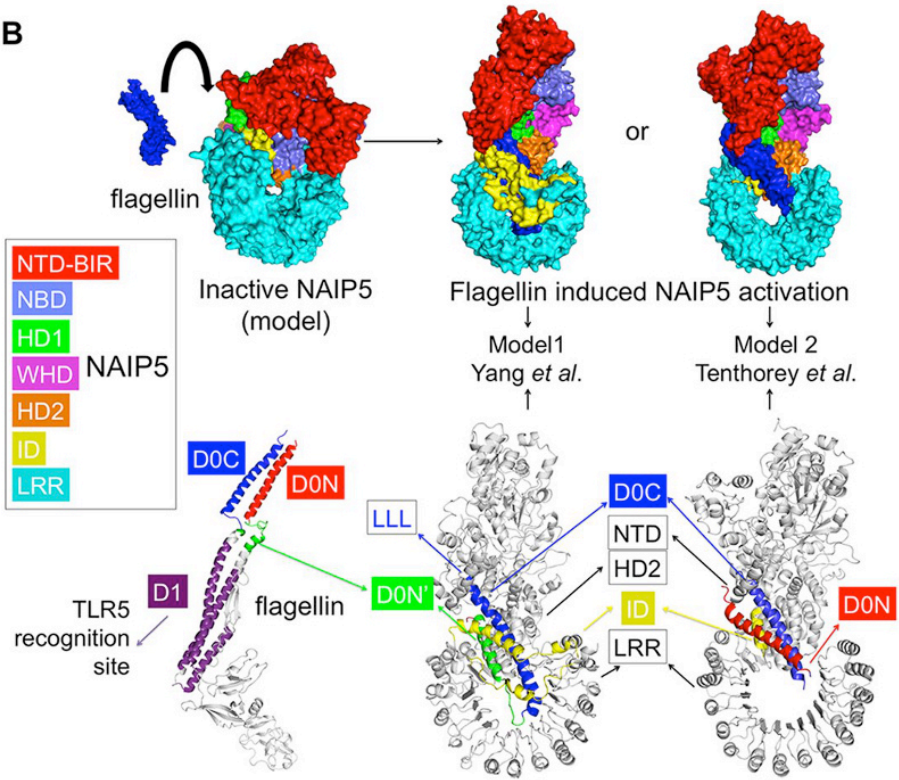

Figure 1 The flagellin/NAIP5/NLRC4 inflammasome structure. (A) NLRC4 activation upon ligand/NAIP5 binding and inflammasome disk formation (PDB: 3JBL). Forest green, NLRC4. (B) NAIP5 activation upon flagellin binding in surface representation (top) and ribbon diagrams (bottom), displaying the two different models by Yang et al. (PDB: 5YUD) and Tenthorey et al. (PDB: 6B5B), respectively. Domains in NAIP5 and flagellin (PDB: 1UCU) are color coded as shown. LLL represents the three important leucine residues on D0C. Flagellin recognition site by TLR5 is shown.

out an exception in which mutation on an extreme C-terminal arginine residue of the D0 domain compromised NAIP5 recognition of flagellin. Strikingly, residues in contact with flagellin are not conserved amongst NAIP5, NAIP2 and hNAIP, which may explain their specificity in sensing different bacterial effector proteins.

The cryo-EM maps reported by these two papers are quite similar and two models traced D0C at the same position, but their interpretations at D0N and NAIP5 ID regions are different. Yang et al. traced the flagellin so that D0N' sits between HD2 and LRR and directly binds NAIP5, whereas D0N does not participate in the binding and was not modeled (Figure 1B, Model 1). How- ever, Tenthorey et al. put D0N to a helix at the surface of the map between NTD and LRR of NAIP5 (Figure 1B, Model 2); this helix was traced by Yang et al. as part of the ID, whereas the ID was not traced in the Tenthorey et al. model.

It is unknown whether the different explanations for the density map is due to the different flagellin species and constructs used by the two groups, however, these differences raised several questions: (1) In addition to the D0C, which part of flagellin is more pathogenic, D0N or D0N'? (2) Does flagellin change conformation upon NAIP5 interaction? The model by Tenthorey et al. requires minimal conformational changes in the flagellin, whereas the model by Yang et al. dictates partial re-folding of the D0N' region. (3) How does the ID domain of NAIP5 function in ligand recognition? (4) What conformational changes do the BIR and ID domains undergo to facilitate ligand binding? A better EM map or more experimental data are needed to fully address these questions.

Interaction of flagellin with NAIP5 is different from flagellin recognition by TLR5 in several aspects: (1) The D1 domain of flagellin is recognized by TLR5 while the D0 domain activates NAIP5 (Figure 1B). (2) Only the LRR domain of TLR5 recognizes flagellin, but a more extensive interaction network is used in the flagellin/NAIP5 complex. (3) Flagellin binding leads to dimerization of TLR 5 to facilitate activation. In comparison, activation of NAIP5 is achieved by a conformational change. With the exciting progress these two papers made, further studies on auto-inhibited conformation of NAIP will give a better understanding about the conformational change of NAIP proteins upon ligand binding, especially in the BIR and ID domains.

\section{Liman Zhang ${ }^{1}$, Hao $\mathrm{Wu}^{1}$}

${ }^{1}$ Department of Biological Chemistry and Molecular Pharmacology, Harvard Medical School, and Program in Cellular and Molecular Medicine, Boston Children's Hospital, Boston, MA 02115, USA

Correspondence: $\mathrm{Hao} \mathrm{Wu}$

E-mail:wu@crystal.harvard.edu

\section{References}

1 Hu Z, Yan C, Liu P, et al. Science 2013; 341:172-175.

2 Kofoed EM, Vance RE. Nature 2011; 477:592-595.

3 Zhao Y, Yang J, Shi J, et al. Nature 2011; 477:596-600.

4 Reyes Ruiz VM, Ramirez J, Naseer N, et al. Proc Natl Acad Sci USA 2017; 114:1324213247.

5 Hu Z, Zhou Q, Zhang C, et al. Science 2015; 350:399-404.

6 Zhang L, Chen S, Ruan J, et al. Science 2015; 350:404-409.

7 Tenthorey JL, Haloupek N, López-Blanco JR, et al. Science 2017; 358:888-893.

8 Yang X, Yang F, Wang W, et al. Cell Res 2018; 28:35-47. 